Recepción: 22/11/2018

Aceptación: 19/01/2019

Publicación: 20/02/2019

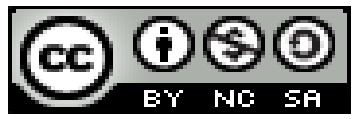

Ciencias de la educación

Artículo de revisión

\title{
Los distintos enfoques en la enseñanza de inglés como lengua extranjera aplicados en "La Unidad Educativa Eugenio Espejo" y "Franquicia Internacional Wall Street English-Loja"
}

The different approaches in the teaching of English as a foreign language applied in "Eugenio Espejo Educational Unit" and "Wall Street English-Loja International Franchise"

As diferentes abordagens no ensino do inglês como língua estrangeira foram aplicadas na "Unidade Educacional Eugenio Espejo" e na "Wall Street EnglishLoja International Franchise"

Andrea Cecibel Campoverde-Castillo ${ }^{\text {I }}$ andrea_cecibel@hotmail.com

Yuraima Yannine de los Ángeles Zambrano-Mendoza II yuraimazambrano1980@gmail.com

Toa Shirin Foorotan-Solano de la Sala III toasfs@gmail.com

\section{Correspondencia: andrea_cecibel@hotmail.com}

\footnotetext{
${ }^{\mathrm{I}}$ Magíster en Orientación y Educación Familiar, Licenciada en Psicología, Docente de la Universidad Nacional de Loja, Loja, Ecuador

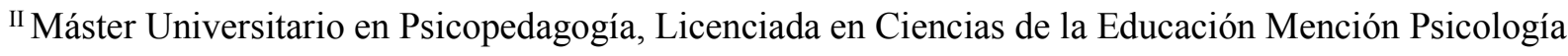 Infantil y Educación Parvularia, Docente de la Universidad Nacional de Loja, Loja, Ecuador

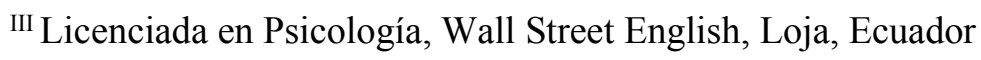




\section{Resumen}

Este estudio presenta los distintos enfoques de la enseñanza de inglés como lengua extranjera aplicados en la "Unidad Educativa Eugenio Espejo" y "Franquicia Internacional Wall Street English-Loja”. Resume las metodologías más utilizadas en la actualidad para la adquisición de una segunda lengua, compara y sintetiza como han sido utilizadas en ambos lugares. Además, señala las diferentes técnicas metodológicas existentes, las mismas que basan sus preceptos en contribuciones de psicología en la enseñanza de un segundo idioma, además destaca diferentes visiones del aprendizaje resumida en procedimientos que han sido utilizados alrededor de los últimos años.

Describe ambos métodos utilizados y la eficiencia que posee cada uno de los mismos. A partir de esta base se han descrito los siguientes métodos: El método de Gramática-Traducción, Audiolingual, la vía silenciosa "Método innovador", respuesta física total, sugestopedia, enfoque comunicativo, conductismo, conectivismo, humanismo, y finalmente estrategias metodológicas didácticas e innovadoras.

Dentro de sus conclusiones, se pudo constatar que la metodología del método blender aplicada en la franquicia internacional WALL STREET ENGLISH-LOJA y los medios alternativos como cuentos, manualidades, juegos, canciones y rimas aplicados en la Unidad Educativa Particular Eugenio Espejo - Escuela, debido a su gran variedad han sido eficaces como herramientas a disposición del docente para fomentar estructuras lingüísticas, vocabulario y conocimientos culturales.

Palabras Claves: metodología; enseñanza; inglés; psicología de aprendizaje; TICS

\section{Abstract}

This study presents the different approaches to teaching English as a foreign language applied in the "Eugenio Espejo Educational Unit" and "Wall Street English-Loja International Franchise". It summarizes the most used methodologies for the acquisition of a second language, compares and synthesizes how they have been used in both places. In addition, it points out the different existing methodological techniques, the same ones that base their precepts on contributions of psychology in the teaching of a second language, in addition it highlights different visions of the learning summarized in procedures that have been used around the last years. 
Describes both methods used and the efficiency of each one of them. From this base, the following methods have been described: The Grammar-Translation, Audio-lingual method, the silent route "Innovative method", total physical response, sugestopedia, communicative approach, behaviorism, connectivism, humanism, and finally methodological strategies didactic and innovative.

Among its conclusions, it was found that the methodology of the blender method applied in the international franchise WALL STREET ENGLISH-LOJA and the alternative media such as stories, crafts, games, songs and rhymes applied in the Particular Education Unit Eugenio Espejo - School, due to their great variety they have been effective as tools available to the teacher to promote linguistic structures, vocabulary and cultural knowledge.

Keywords: methodology; teaching; English; learning psychology; TICS

\section{Resumo}

Este estudo apresenta as diferentes abordagens para o ensino do inglês como língua estrangeira aplicada na "Unidade Educacional Eugenio Espejo" e na "Wall Street English-Loja International Franchise". Ele resume as metodologias mais utilizadas para a aquisição de uma segunda língua, compara e sintetiza como eles foram usados em ambos os lugares. Também chama a atenção para as diferentes técnicas metodológicas existentes, eles baseiam seus preceitos sobre as contribuições da psicologia no ensino de uma segunda língua também destaca diferentes visões de aprendizagem descrito nos procedimentos que têm sido usados em torno dos últimos anos.

Descreve os dois métodos utilizados e a eficiência de cada um deles. A partir desta base que foram divulgados os seguintes métodos: O método gramática-tradução, lingual áudio, forma silenciosa "Método inovador" resposta física total, sugestopedia, comunicativo abordagem conductismo, conectivismo humanidade, e finalmente estratégias metodológicas didático e inovador.

Entre as suas conclusões, verificou-se que a metodologia do liquidificador método aplicado no franchising internacional WALL STREET meios alternativos como histórias, artesanato, jogos, canções e rimas usados na Private Education Unit Eugenio Espejo Inglês-LOJA e - Escola devido para sua grande variedade, foram eficazes como ferramentas disponíveis ao professor para promover estruturas lingüísticas, vocabulário e conhecimento cultural.

Palavras-chave: metodologia; ensino; Inglês psicologia da aprendizagem; TICS 


\section{Introducción}

Los distintos enfoques en la enseñanza de inglés como lengua extranjera, se encargan de buscar métodos y técnicas con el fin de mejorar la enseñanza, procurando que los conocimientos lleguen de una forma eficaz a los educandos. Así mismo, se entiende a aquella disciplina de carácter científico-pedagógica que se focaliza en cada una de las etapas del aprendizaje.

Desde la antigüedad, la importancia de la comunicación ha representado poder y conocimiento. Reyes, reinas, emperadores, mercaderes, navegantes, soldados; una lista interminable de personas, han tenido la necesidad de hablar y comunicarse en otros idiomas. Cada uno con diferentes motivos, empujados por la necesidad de entender y ser entendidos en un idioma diferente. Sin embargo, los estudiosos consideraban que el lenguaje oral no merecía la pena estudiarse, y por tanto no establecieron reglas ni patrones correctos, luego en el siglo XVII, los alumnos traducían frases de sus libros de texto al idioma extranjero, posteriormente leían sus respuestas en voz alta. Esté método les daba únicamente unos minutos de practica oral, sin reglas gramaticales. Así que, fue imposible ganar fluidez verbal o destreza en el uso del idioma, como lo describe Hearn (2006).

La adquisición de un segundo idioma, depende de diversos factores, tales como: culturales, sociales, profesionales o personales. Este objetivo se consigue mediante la integración de contenido, práctica y la relación de inmersión estudiante-idioma. En la investigación denominada "Metodología aplicada en el curso de idioma inglés ciclo de educación básica en los institutos oficiales del Municipio de Morales en el departamento de Izabal" escrita por Calderón (2006). Se postula distintas metodologías de enseñanza del inglés, entre las más conocidas tenemos: El método de Gramática-Traducción (G-T), el método directo, el método Audio-lingual, la vía silente de Gattegno, el método de respuesta física total (TPR) y el enfoque comunicativo.

\section{Desarrollo}

El método de Gramática-Traducción (G-T): dentro de la enseñanza es el más antiguo, parte de las escuelas de latín; sin embargo, en lo posterior se utilizó para la instrucción del idioma inglés. Según Calderón (2006). Este método está basado en la gramática y presta atención a: Reglas gramaticales, lista de vocabulario y ejecución de ejercicios de traducción. Estos elementos fueron 
relevantes e incluso se utilizaron como técnica principal para la explicación de nuevas estructuras gramaticales. Este aporte permitió que se incluyeran nuevas palabras, creando un camino directo para la adquisición de la lengua en general. En este método se utilizaba una oración en la lengua madre, para que la misma pueda ser traducida literalmente a la lengua extranjera. Con esta práctica se pretendía analizar sus componentes gramaticales en términos de la gramática de la lengua madre a partir de la creencia errónea de que los patrones gramaticales son universales y aplicables a cada una de las distintas lenguas. De esta manera, no se ejercitaba de forma adecuada las habilidades auditivas, orales y no se desenvolvía el pensamiento en la lengua extranjera, como lo explica en su estudio Calderón (2006).

Con el tiempo y de forma acertada se van definiendo nuevas metodologías, potenciadas por el uso de tecnologías físicas y virtuales que se combina en el diseño académico. Mejorando de esta manera el proceso de aprendizaje (Martínez2018).

Desde siempre se ha evidenciado la necesidad de analizar el proceso de aprendizaje con una perspectiva multidisciplinar que articule la praxis docente y el desenvolvimiento académico de los estudiantes y que fortalezca los componentes teóricos - prácticos del aprendizaje, demostrando la coexistencia de la tradición gramatical y conversacional en la enseñanza de lenguas. Es por ello que es aplicable como una reacción al método Gramática Traducción. Este método destaca por su practicidad, y “deviene del hijo menor del método blended o también conocido como natural, psicológico, fonético y de lectura (Calderón 2006). A partir del siglo XX, surgen nuevos cambios sociales y tecnológicos, nace la era de la industrialización y expansión comercial. Estos elementos constituyeron el pre-requisito para el resurgimiento de este método. (Sánchez, 1997).

Su denominación como "directo" expresa que la palabra extranjera debe conectar de forma directa con las acciones, gestos y situaciones, sin hacer uso de la lengua materna, como lo explica Mato (2011), en su investigación: "Principales métodos de enseñanzas de lenguas extranjeras en Alemania". Esta metodología sugiere el uso de diversas técnicas incentivando al docente a relacionar palabras con gráficos. Otra aportación, a esta metodología básica, consiste en el desarrollo de la habilidad ORAL, asumiendo que el aprendizaje de la lengua extranjera y la lengua materna constituyen procesos similares. Este método conductor, favoreció al 
esclarecimiento en el aprendizaje de las lenguas. Resaltando que es más relevante la actividad psicológica, que el uso de la lógica. Este enfoque es importante para generar el interés y la atención del estudiante. Obteniendo resultados favorables en el aprendizaje.

Según Mato (2011), el método Audio-lingual asocia imágenes con palabras mediante ejercicios de repetición. Se enfoca en ejercicios mecánicos e imita patrones nativos. Evidentemente, Skinner y el neo-conductismo, tratan la adquisición de una lengua extranjera como un acumulo de hábitos y lo toma como una forma conducta social ante el medio. Para Skinner, la adquisición de una lengua extranjera, se postula como forma de expresión natural. Por ello no podrá desarrollar todas las habilidades tal como un entendimiento de los hablantes nativos.

Cabrera (2014), describe el estudio del método innovador, cuyo propósito es la construcción de la autoconfianza, basado en que, el tiempo de habla del docente debe ser reducido o disminuido, pero motivar a los estudiantes a que generen la producción mayoritaria del lenguaje hablado en la clase. Por otro lado, Mato (2011) postula sobre el rol del docente como facilitador quien provee de ayuda en diversos momentos siempre y cuando el estudiante presente algún inconveniente. El proceso de enseñanza aprendizaje se basa en sus sonidos asociándolos con colores. Siendo la corrección de errores la evidencia de donde la lengua no está clara y de esta manera reforzar las áreas donde se debe enfocar y priorizar.

Además, se puede nombrar el método de Respuesta física total, cuyo objetivo es el divertirse aprendiendo. De esta manera el aprendizaje, según Silvia (2018), se da por medio de la misma metodología de aprendizaje de su lengua materna, utilizando un ciclo natural de aprendizaje. El aprendizaje se da por medio de la actividad física motora. El rol del docente consiste en la dirección del comportamiento de los estudiantes e imitar. Por otro lado, el método de Sugestopedia, el cual se originó en los años 60 y parte de la idea de explotar las habilidades del ser humano. La sugestopedia intenta llegar a explotar la autodisciplina basándose en disciplinas terapéuticas como la psicoterapia comunicativa.

Finalmente, se presenta el enfoque comunicativo, el cual prioriza las intenciones comunicativas, dejando en un papel secundario a las estructuras gramaticales. Esta metodología toma las ideas básicas de cada método descrito con anterioridad y la combina logrando de esta manera la 
adquisición de una competencia comunicativa. Este enfoque según Richards (1986), ha sido construido como una reacción a los métodos gramaticales o de traducción (Mato, 2011). En la práctica, éste método manifiesta cognitivismo y ya que como objetivo final humanismo busca la comprensión como competente básico y el desarrollo polifuncional de cada una de las habilidades. Se asimila al método gramática-traducción, pero como una creación más sofisticada y acabada, aunque este enfoque trata de integrar patrones de pensamiento propios de los cognitivistas con la afectividad y empatía del humanismo (Calderón 2006).

Si bien se ha descrito los fundamentos y estructura de los métodos debería describir la fundamentación teórica, los modelos psicológicos que entran en debate en este tipo de metodología tomadas de la investigación, "Blended learning. Conceptos básicos. Pixel-Bit”. Que presento Bartolomé. (2015).

Conductismo: Por medio de la plataforma virtual multimedia, los estudiantes realizan ejercicios y prácticas repetitivas con corrección automática y presentación de respuestas correctas, ejercitación y práctica, presentaciones visuales con continuo feed-back y retroalimentación positiva, por ende, el aprendizaje es específico debido a que ellos mismos pueden evidenciar el error y asociar sus falencias para mejorarlas.

Cognitivismo: El tipo de información que el estudiante recibe es por medio de software que facilita la exploración de contenidos e indagación sobre los mismos, lo cual permite que el estudiante desarrolle las habilidades y destreza al organizar la información proporcionada por medio de los esquemas cognitivos que articula el aprendizaje a través del trabajo de la memoria. Humanismo: La presentación de las clases se las realiza de forma individual y enfocándose en las necesidades de cada uno de los estudiantes, basadas en las destrezas de atención y diferencias pág. 10 individuales para el trabajo colaborativo, tomando en consideración las inteligencias múltiples planteadas por Gardner y los estilos de aprendizajes tomados por Vark(2013).

\section{Metodología}

La metodología utilizada fue de tipo descriptivo - comparativo, en tanto que permitió resumir las metodologías más utilizadas en la actualidad para la adquisición de una segunda lengua, así como comparar y sintetizar como han sido utilizadas en ambos centros educativos, sometidos a investigación. La población la constituyeron aprendices del idioma de cada una de las 
instituciones evaluadas. Los resultados se presentan posterior a la aplicación de cada una de las metodologías de enseñanza del inglés como lengua extranjera.

\section{Resultados}

\section{Aplicación de la Metodología de la enseñanza de inglés como lengua extranjera de la franquicia internacional Wall Street English.}

Después de doce meses de experiencia laborando en la franquicia internacional WALL STREET ENGLISH-LOJA, se ha podido determinar que es un método nuevo y combinado. El método de enseñanza que utiliza esta prestigiosa marca se denomina “BLENDED LEARNING METHOD”. El diseño metodológico se centró en el estudiante como foco directo. Además de optimizar el tiempo debido a la combinación de una plataforma interactiva virtual y clases presenciales. De esta manera este concepto recibiría otra denominación catalogándolo como "educación flexible o modelo hibrido" (Martínez, 2018).

Durante el recorrido profesional se ha logrado estar en contacto con diversas metodologías, e incluso se ha impartido clases utilizando varias de ellas. En cada una de esas clases impartidas se ha visualizado que el aprendizaje de un nuevo idioma es un desafío para los aprendices, sin embargo, con las herramientas adecuadas se puede hacer de este proceso entretenido y ventajoso. Wall Street English ofrece algo distinto y novedoso, trabaja con grupos pequeños y clases personalizadas y guiadas por un profesor certificado internacionalmente con el dominio de la metodología "Blended Learning Method".

Las planificaciones se encuentran previamente elaboradas es decir el docente solo debe ejecutar lo escrito, el tiempo de explicación del profesor es reducido de total de los 60 minutos de clases impartidos tan solo el $15 \%$ es producción de los docentes clasificándolo más como un facilitador del idioma.

La producción de la clase es dada en su mayoría con el $85 \%$ dictada por el estudiante debido a que son clases pre-diseñadas, el aprendiz por medio de una plataforma interactiva y siguiendo el método natural de adquisición de habilidades (Escuchar, pronunciar, practicar, hablar, confirmar, leer y escribir) desarrolla su clase y entra en un proceso de inmersión con el idioma. En este 
método de aprendizaje el uso de las TICs es prioritario para que los estudiantes procesen una cantidad cada vez mayor de información (Martínez, 2018).

La parte multimedia y la plataforma virtual interactiva de aprendizaje toma lo mejor de las distintas metodologías; como del método de Gramática-Traducción, Audio-lingual, La vía silenciosa "Método innovador", de la respuesta física total, sugestopedia y del enfoque comunicativo y las combina creando de esta manera una plataforma completa donde el estudiante puede poner en práctica cada una de las habilidades del idioma y la adquisición del idioma lo realiza en un tiempo menor (Paz, 2017).

\section{Aplicación de la Metodología de la enseñanza de inglés como lengua extranjera en la unidad educativa particular Eugenio Espejo- escuela en Loja Ecuador.}

Tomando en cuenta que la educación dentro de la unidad educativa Eugenio Espejo va dirigida a niños entre las edades de 5 a 12 años y el aprendizaje de los niños que se da por medio de las inteligencias múltiples presentadas por Gardner como la verbal-lingüística, cinética-corporal, lógica-matemática, visual-espacial, inteligencia naturalista, inteligencia interpersonal e inteligencia intrapersonal o por medio de los estilos de aprendizaje de Vark (2013), quien considera, que un aprendizaje significativo se provee por medio de los cinco sentidos. Por tal razón, es importante mencionar la aplicación de diversos medios y recursos en la enseñanza del inglés como segundo idioma tales como:

Trabajar con Cuentos: Enfocando y desarrollando la imaginación de los pequeños, llevándolos a un viaje por un mundo desconocido y llamativo captando su atención e interés por el idioma.

Desarrollar manualidades: por medio de las mismas se trabaja con los alumnos que tienen un sistema de aprendizaje cinético corporal con el fin de que la dinámica de enseñanza sea interactiva.

Incrementar juegos: Este modelo es muy importante ya que se ha demostrado que la actividad física y mental ayuda al aprendizaje de los alumnos, desde su sensibilidad de niños, para que se sientan considerados y valorados.

Aumentar el uso de Chants, estos facilita el aprendizaje y tiene como objetivo incrementar el vocabulario de los pequeños. 
Desarrollar el Role Play, como una forma útil de incorporar a los estudiantes una educación activa.

Generar proyectos dentro de la institución con el apoyo familiar, este plan de trabajo es muy importante porque se expone lo aprendido dentro del aula.

Incorporar a los padres en el desarrollo de tareas en familia. Tomando en cuenta que su rol es fundamental para reforzar el estudio y práctica en el aprendizaje de sus hijos.

Incentivar la cultura tecnológica por medio de películas que contribuyan a desarrollar la imaginación de los pequeños.

Fomentar la cultura mediante las actividades prácticas y los recursos didácticos como textos y guías, previamente elegidos por la institución para obtener buenos resultados y que el aprendizaje del inglés como segundo idioma sea representativo.

Dentro de la institución se consideró realizar actividades extracurriculares, por medio de un “contest" llamado cantando en inglés. Invitando a la participación a otras instituciones educativas de la ciudad.

\section{Conclusiones}

La metodología aplicada tales como: método blender aplicada en la franquicia internacional WALL STREET ENGLISH-LOJA, los medios alternativos como cuentos, manualidades, juegos, canciones y rimas aplicados en la Unidad Educativa Particular Eugenio Espejo - Escuela, debido a su gran variedad ha sido eficaz, ya que son herramientas a disposición del docente que fomentan estructuras lingüísticas, vocabulario y conocimientos culturales.

Los procesos antes señalados se consideran factibles al momento de enseñar el idioma inglés, los mismos que fueron evaluados cualitativamente por medio de aprendizaje colaborativo como: entrevistas, proyectos expuestos por los estudiantes en donde se pudo observar una fluidez pág. 12 y correcta comprensión del idioma, además de evidenciar el aprendizaje en los estudiantes de manera integral, efectiva, social y artística.

La creatividad mediante los proyectos aplicados en la Unidad Educativa Particular Eugenio Espejo - Escuela, desde un enfoque, sirven a los docentes con el fin de conectarse profesor- 
alumno debido a la eficacia en la búsqueda de información y cooperación en donde el estudiante aprende de una manera multidisciplinar.

Se identificó que, tanto los medios tecnológicos, como los variados recursos metodológicos que los docentes proseen, son muy importantes para lograr los objetivos. Con el fin de obtener un efectivo proceso de enseñanza aprendizaje.

\section{Referencias Bibliográficas}

Hearn, I (2006). Didáctica del Inglés. España: Pearson Prentice Hall.

Cabrera, H. (2014). Historia de las ciencias en la enseñanza de las ciencias: el caso de la reacción química. Revista Brasileira de Historia Da Ciencia, 7(2), 298-313

Vark, H (2013). Teoría de Vark. Fundación Ver Bien Para Aprender Mejor. Recuperado de: http://verbien.org.mx/teoria-de-vark

Association, P. (1991). Publication Manual of the American Psychological Association.

Bartolomé, A. (2015). Blended Learning conceptos básicos. Universidad de Barcelona, 5-8.

Calderón, M. (2006). Metodología Aplicada en el curso de idioma Ingles, ciclo d educación básica en los institutos oficiales del Municipio de Morales departamento de Izabal. Guatemala: Universidad de San Carlos de Guatemala.

Martínez, D. A. (2018). BLENDED LEARNING: Modelo presencial-virtual de aprendizaje y aplicación en entornos educativos. UNIVERSIDAD DE ALICANTE, 1-3.

Mato, N. (2011). Principales Métodos de Enseñanza de Lenguas Extranjeras en Alemania: Revista de lingüística y lenguas aplicadas.

Paz, A. M. (2017). Enseñanza del inglés a través de las apps. Universidad de Cantabria, 7-8.

Richards, J. (1986). Enfoques y métodos en la enseñanza de idiomas. Madrid: Cambridge Univesity Press.

Sánchez, A. (1997). Los métodos en la enseñanza de idiomas. Evolución histórica y análisis didáctico. Madrid: SGEL.

Silvia, B. (2018). Los distintos enfoques en la enseñanza de inglés como lengua extranjera y segunda: nuevas tendencias en la literatura contemporánea. Revista de educación, 101102. 